\title{
Current advances in the basic research and clinical management of sinonasal inverted papilloma (Review)
}

\author{
ALEXANDER SAUTER $^{1}$, RUBINA MATHARU ${ }^{2}$, KARL HÖRMANN $^{1}$ and RAMIN NAIM ${ }^{1}$ \\ ${ }^{1}$ Department of Otolaryngology, Head and Neck Surgery, University Hospital Mannheim, \\ D-68135 Mannheim, Germany; ${ }^{2}$ Warwick Medical School, University of Warwick, Coventry, UK
}

Received September 18, 2006; Accepted November 24, 2006

\begin{abstract}
Inverted papilloma (IP) is a benign sinonasal lesion that has a known propensity for recurrence, local aggressiveness and an association with transformation to squamous cell carcinoma. Due to the high rate of recurrence, association with malignancy and a tendency of multicentricity, the surgical approaches to treatment are controversial. Over the years there has been a slow evolution from aggressive (en bloc) resection by lateral rhinotomy to endoscopic techniques. This progress corresponds to the advances that have been made in endoscopic sinus surgery over the past 15 years. Technological advances have allowed the detection of sinonasal IP before its extension beyond the sinonasal region, thus enabling minimally invasive techniques to be used in the treatment of selected cases of IP. Differences in recurrence rates were not observed for endoscopic management as compared with lateral rhinotomy or sublabial degloving approaches. In terms of aetiology there is certain evidence that the presence of HPV in IP could be predictive of malignant transformation. Although IPs are monoclonal proliferations, they do not fit the profile of a prototypic precursor lesion. In contrast, an increased EGFR and TGF- $\alpha$ expression is associated with early events in IP carcinogenesis. Parameters such as hyperkeratosis, squamous epithelial hyperplasia and a high mitotic index are negative prognostic indicators, which could be useful in the future follow-up of patients with IP. Present literature should encourage us to recommend the use of a uniformly accepted staging system. The propensity for delayed recurrences and the maximal $13 \%$ incidence of malignant transformation mandates careful, long-term follow-up.
\end{abstract}

Correspondence to: Dr Alexander Sauter, Universitäts-HNO-Klinik Mannheim, Theodor-Kutzer-Ufer, D-68135 Mannheim, Germany E-mail: alexander.sauter@hno.ma.uni-heidelberg.de

Key words: inverted papilloma, p53, human papilloma virus, EGFR, TGF- $\alpha$, paranasal sinuses, nose, endoscopic sinus surgery, surgical procedures, recurrence rates

\section{Contents}

1. Epidemiology and aetiology

2. Histopathology

3. Cytogenetics and molecular genetics

4. Classification

5. Macroscopic aspects

6. Surgical advances

7. Conclusion

\section{Epidemiology and aetiology}

Inverted papilloma (IP) is a lesion of the mucosal membrane of the nasal cavity and paranasal sinus. It is a benign epithelial growth in the underlying stroma that has also been referred to as villiform cancer, Schneiderian papilloma, transitional cell papilloma, cylindrical cell papilloma, papillary sinusitis, and Ewing's papilloma (1). IP is a benign sinonasal tumor of ectodermal origin which is locally destructive and has a tendency to recur if incompletely removed. In addition, it has a significantly malignant potential.

Ward (2) and Billroth (3) were the first to describe IP as a defined lesion but it was not until much later that Kramer and Som distinguished the pathology from simple polyps (4). Ringertz revealed microscopically the behaviour of IP in invading the underlying stroma but still felt that it was in some way associated with simple nasal polyposis (5). We now know that IP is a distinct pathology with a characteristically clinical process. Since the early 1980 s research has attempted to resolve the question of deciding between conservative (endonasal) management and more radical treatment in the way of lateral rhinotomy with medial maxillectomy.

IP is a rare tumor occurring in $\sim 0.5$ to $7 \%$ of all the cases of nasal tumors, thus representing $\sim 4 \%$ of all nasal polyps and $\sim 70 \%$ of all the cases of sinonasal papilloma $(6,7)$. The incidence of IP has been estimated at 0.74/100,000/year (8). There is a male predominance of 2-4:1 and White Caucasians are more likely to be affected than those who are of AfroCaribbean origin (9).

Most patients present with this pathology in their sixties, the average age at presentation being 53 (6). Isolated observations in the paediatric and adolescent group have also been reported $(10,11)$. 
The typical presentation is of unilateral polyps. Differential diagnoses include an antral choanal polyp, allergic fungal sinusitis, squamous cell carcinoma, adenocarcinoma, esthesioneuroblastoma, IP and other rare tumors.

Clinical symptoms were, initially, unilateral nasal obstruction in the majority of the presented cases, followed by epistaxis, clear rhinorrhea, post nasal drip and a feeling of pressure. Facial pain, hyposmia, anosmia, and epiphora were rarely encountered. The most frequent symptom of nasal obstruction, was observed in 80 to $98 \%$ of the patients $(12,13)$. In patients with simultaneous association of IP and malignancy, epistaxis was the most common symptom (12). Associated sinusitis indicated by pus evident at the time of resection occured in 34 to $39 \%$ of patients $(12,15)$.

The mean duration of the symptoms was from 7.2 months to 26 months before hospital admittance. However, some authors also observed patients with a history of symptoms of up to 5 years with no obvious progression of tumor growth $(13,14)$.

Although the aetiology of IP is still unknown, recent studies using in situ hybridization and polymerase chain reaction have detected human papilloma virus (HPV) in up to $86 \%$ of IPs (16). The presence of HPV DNA in sinonasal papilloma was reported for the first time in $1987(17,18)$. In particular, the viral subtypes $6,11,16$, and 18 were the most frequently found (19-21). The current advances in viral investigations in IP will be presented in the continuation of this review.

Environmental pollutants such as cigarette smoke, are also likely to be significant. It has been shown that cigarette smoking increases the risk of progression to squamous cell carcinoma (SCC) in IP (22). Allergy, chronic sinusitis, and other chemical pollutants have also been suggested as possible causes $(6,23,24)$. Allergy is unlikely however, since most of the patients do not have an allergic history and the polyposis associated with allergic rhinitis is usually bilateral. The presence of sinusitis is related more to the obstructive nature of the disease than the cause. The signs of chronic infection of the sinuses are much too common to be implicated in the rare case of IP.

The presence of pre- or coexisting IP is a well-known risk for the development of sinonasal cancer. A distinction between 3 different types of papillomas arising in the respiratory epithelium has to be made: i) Exophytic papillomas (ICD-O 8121/0), arising mainly in the nasal vestibule rather than in the paranasal sinuses, almost never exhibit malignant transformation. There is only 1 reported case of such malignant transformation (25). ii) IPs however, (ICD-O $8121 / 1$ ), arising primarily in the paranasal sinuses show malignant transformation or coexisting carcinomas in $0 \%$ (26-29) to $53 \%$ (30) in certain studies with great variations in the numbers of treated patients. iii) Columnar cell papilloma (cylindrical cell papilloma, ICD-O 812171) is the least common type of nasal papilloma. It is known that this type of papilloma can undergo malignant transformation, as shown in a study by Kapadia et al (31). With very few exceptions, IP and sinonasal malignancy are not an inherited disease. We found only one reported case of recurrent IP, in which inheritance could be suspected (11).

\section{Histopathology}

IP mainly looks like a polyp with the exception that it is usually firmer and significantly bulkier, with a more granular mulberry-type appearance. It can range from a variery of shades from red to pale pink. They are generally more vascular than the average polyp. Microscopically the lesion has a thickened epithelial multilayer which covers an extensive invasion of the hyperplastic epithelium into the underlying stroma. There are often goblet and columnar respiratory elements admixed $(5,32)$. The behaviour of the invasion into the underlying stroma has been theorised to be due to an origin from the Schneiderian membrane (Fig. 1).

The Schneiderian membrane is of ectodermal origin from the nasal placode and certain differences in the underlying stroma could permit the inversion of the papilloma. The tumor has crypts which are subepithelial and maintain a connection to the surface epithelium at all times, a finding which lead to the name inverted papilloma. Mucus containing microcysts is often trapped within the neoplastic epithelium. The covering epithelium can be squamous, respiratory, transitional cell epithelium or a combination of the three. The cells show minimal nuclear atypia with the typical basilar layer mitosis. The stroma usually has both acute and chronic inflammatory changes with areas of fibrosis and oedema. The stroma is almost lacking in eosinophils which would be prevalent in an allergic polyp.

IP typically comprises both exophytic and endophytic components (32). The tumor invaginates or infolds into the surrounding underlying bone. However, it does not invade in the absence of malignancy. To an inexperienced pathologist a specimen which is tangentially cut could lead to the misinterpretation that the epithelium is not connected to the surface, simulating stroma invasion.

The incidence of focal malignancy within IP or a site adjacent to the papilloma ranges from 0 to $53 \%$ (26-30). An association between IP and SCC as high as 53\% is likely to be overestimated. Data from the largest studies $(7,11,33)$ and recent accurate reviews of the literature $(15,34)$ indicate that the two diseases are concomitantly diagnosed in 3 to $13 \%$ of the patients. A further 1 to $1.5 \%$ of patients have been shown to present a metachronous malignant lesion $(35,36)$. The development of carcinoma at the site of a previously removed IP is a less common event. In the malignant areas of IP the squamous epithelium shows marked atypia, an increased nuclear to cytoplasmic ratio, conspicuous nucleoli, atypical mitosis in the middle and upper layers, a loss of polarity and dyskeratotic cells. The loss of polarity, anaplasia of the cells and the lack of maturation are the most reliable criteria (37). There is no clearly defined criteria for borderline cases of marked dysplasia or carcinoma in situ. The type of epithelium or stromal inflammation has no role in determining which lesion represents malignancy.

\section{Cytogenetics and molecular genetics}

Viral investigations have been performed in search for an aetiology. Two types of DNA viruses are of interest in malignant transformation: HPV and EBV. The presence of HPV DNA in sinonasal papilloma was reported for the first 


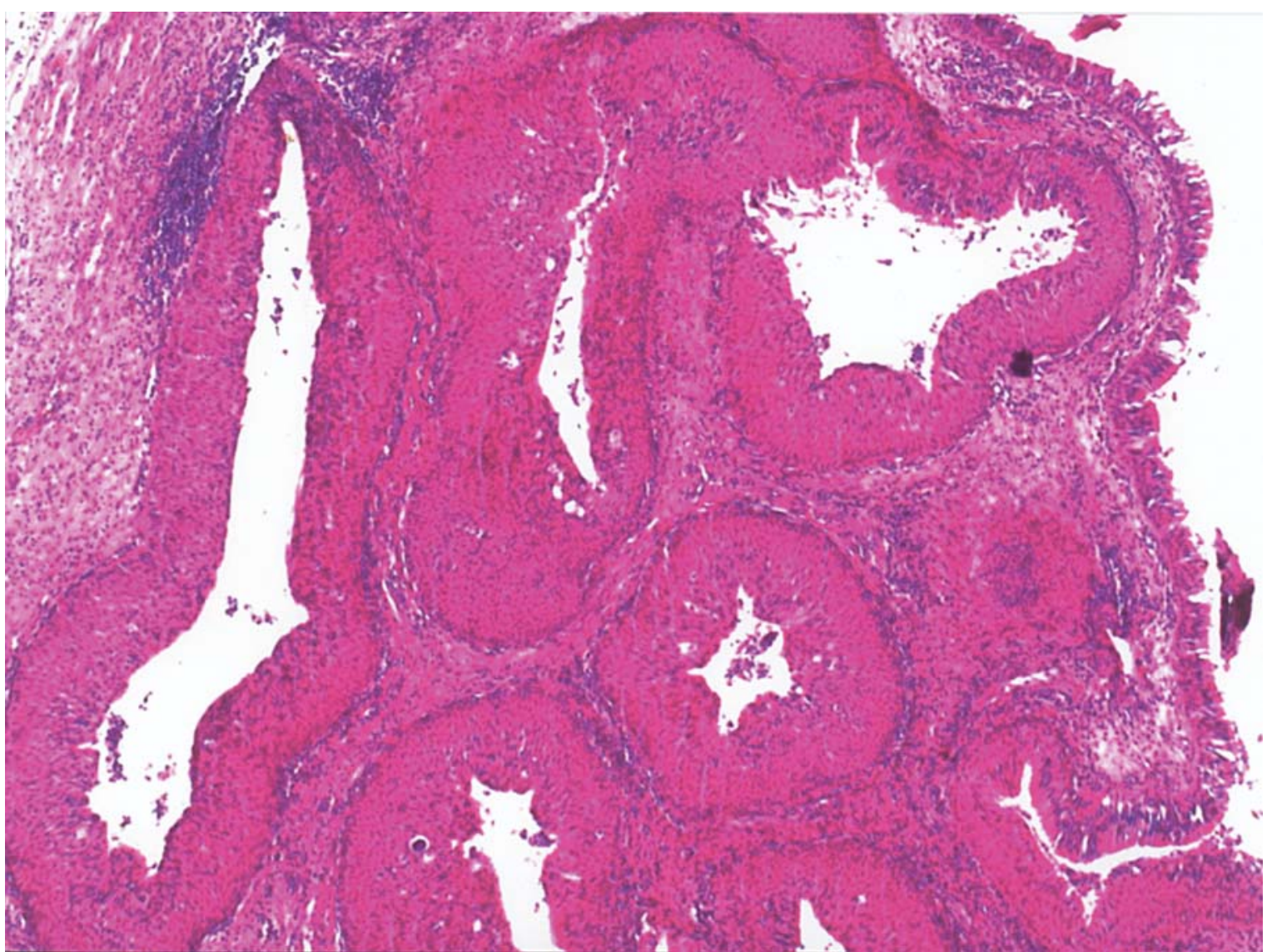

Figure 1. Inverted papilloma of the paranasal sinus showing a thickened epithelial multilayer which covers an extensive pseudoinvasion of the hyperplastic epithelium into the underlying stroma. H\&E staining. (x50)

time in $1987(17,18)$. Several studies have been performed and the results are mixed with the range of HPV DNA present varying from 0 to $100 \%(17,18,38)$ in the IP specimens. Recent studies demonstrate that HPV could be associated with $33 \%$ of IP (39). In over 90 types of HPV that are known to date, only a few are linked with malignant transformation. While HPV types 6 and 11 are detected in the majority of exophytic papillomas of the entire upper respiratory tract (40), IPs contain these HPV types in only $6-8 \%$ of cases (41). Recent studies using in situ hybridization detected HPV 6 and 11 in $42 \%$ of cases. In particular, IP associated with severe dysplasia or carcinoma and HPV 6 or 11 was present in 57 and $67 \%$ of tumors (42). The HPV types associated with malignant transformation are, among others, HPV 16 and HPV 18. Hwang et al detected HPV 16 DNA in 2 out of 5 IPs with coexisting SCC (41), and Kashima et al detected HPV 18 DNA in 1 out of 24 SCCs (43). Katori et al presented HPV 16 or 18 DNA in $31 \%$ of IP tumors and in 42 and $50 \%$ of IPs with severe dysplasia/carcinoma (42). Certain studies have indicated that real-time PCR is more sensitive than Southern blot hybridization in the detection of HPV DNA (44). Using quantitative real-time PCR to evaluate the state of the HPV genome (episomal or integrated) McKay et al presented an association of HPV with IP in $21.4 \%$ or cases (45). In particular, it was detected in two of three patients in which IP was associated with sinonasal SCC. Real-time PCR has shown that in two of three lesions in which IP is associated with SCC, the integration of the HPV genome into the host genome has occurred. HPV E6 and E7 oncoproteins are capable of functionally inactivating cell cycle regulators such as p16, p21, p27, p53, the retinoblastoma gene product $(\mathrm{Rb})$ and cyclin D1 $(46,47)$. Thus, these oncoproteins have the ability to deregulate the cell cycle G1/S transition. Malanchi et al showed that HPV16-E6 drives $\mathrm{p} 16^{\mathrm{INK} 4 \mathrm{a}}$ or $\mathrm{p} 27^{\mathrm{KIP} 1}$ overexpressing cells into the $\mathrm{S}$ phase in rodent immortalised fibroblasts (48). Viral oncoproteins can therefore bypass the negative signals exerted by $\mathrm{p} 27^{\mathrm{KIP} 1}$. Zerfass-Thome et al demonstrated that HPV16-E7 oncoproteins are capable of a direct interaction with $\mathrm{p} 27^{\mathrm{KIP} 1}$. Thereby its association with the cyclin/cdk complexes is inhibited (49). Affolter et al detected high p 27 protein levels in $71 \%$ of an IP cohort (50). These high levels of p27 could be an attempt of the cell to prevent the transition into the $\mathrm{S}$ phase by overexpression of the cell cycle inhibitor. However, there was no significant association between local recurrence and the p27 ${ }^{\mathrm{KIP} 1}$ expression level: Seventy-five percent of the tumors with recurrence and $68.8 \%$ of the samples without recurrence showed an overexpression of the protein (50). Saegusa et al identified weak to strong p27 immunoreactivity in inverted and exophytic papillomas. The author demonstrates that the loss of the p27 expression correlates with the increase of cell proliferation in sinonasal tumors (51). Affolter et al investigated a largely normal to an overexpressed p53 expression status in IP samples which could be associated with HPV infection (50). The mechanisms 
of action of HPV in oncogenesis have been attributed to its ability to render the p53 tumor suppressor gene of the host cell ineffective. The mutation of the p53 gene is currently the most commonly identified gene mutation in human neoplasia (52).

\section{Classification}

Although the last five years have brought some important advances in the classification of IP, the classification of tumors at this anatomical site is still problematic: The anatomical site is complex, and the incidence is low, therefore reliable statistical data on prognosis is still scarce. The classification of malignant tumors by the Union Internationale contre le Cancer took place in 2002 (53). This classification applies to carcinomas of the maxillary sinuses and the ethmoid sinuses and nasal cavity. There is still no widely accepted classification for carcinomas located in the sphenoid and frontal sinus. The practical, clinical and prognostic use of the $\mathrm{T}$ classification for tumors in this region is still controversial, and alternative classifications have been proposed in the past few years (54). The most widely applied system is the one described by the American Joint Committee on Cancer (55). In this system, commonly described as the TNM (tumor, node, metastasis) system, the extent and location of the tumor are reflected in the four $\mathrm{T}$ stages, with the $\mathrm{T} 1$ lesions being the smallest, and $\mathrm{T} 4$ the most contained and more extensive ones. T1 malignancies of the nose and sinuses are confined to one discrete location without any bone involvement. T4 lesions are more extensive, with penetration through the bone and into contiguous regions such as the intracranial compartment.

A similar system that also has practical utility was described by Carinci et al (56). In this approach, malignancies confined to one site are considered $\mathrm{T} 1$ lesions and tumors that extend into separate regions, including the orbit and cranium, are staged as T4. Intermediate-stage lesions extend from the nose into the sinuses or involve more than one sinus cavity. Both of these systems of staging malignancy share two characteristics. They both describe a serial staging system, with confined disease staged as $\mathrm{T} 1$ and intermediate tumors staged as T2 and T3 based on the extent of involvement. In addition, they both stage the extensive spread of disease into structures outside the confines of the sinuses as T4 lesions.

Staging systems have also been developed for use in patients with chronic sinusitis. Besides the five-stage system described by Friedman et al (57), Lund and MacKay outlined a rating score with a range of 0 to 24 (58). In this system, each of the six anatomical locations is graded for the absence of disease, partial involvement, or complete opacification. The locations are each rated bilaterally.

Several authors have developed staging systems specifically for use with IP, although none have been applied in any systematic manner. The first of such systems was described by Skolnick et al (59), who applied the tumor component of the TNM system for staging malignancies of the nose and sinuses. Similar to the staging noted in the AJCC system, the authors described T1 lesions as those confined to one anatomical site within the nose and $\mathrm{T} 2$ lesions as involving two sites within the nose. They staged any involvement of the sinuses as T3, with an extension outside the nose and sinuses as T4. A similar approach was also described by Norris (60). Schneider (61) described a second staging system for IP in which the radiological appearance of the tumor was considered in determining the clinical stage. He argued that the AJCC staging system was not appropriate for use with these benign lesions. In Schneider's system, stage I was described as a tumor confined to the nasal cavity, with stage IV demonstrating erosion through the bone and extension into the dura or periorbit. Intermediate stages demonstrated progressive involvement into the sinuses from the nose.

A third system for staging IP was described by Schwab et al (62). In this system, which is similar to the one proposed by Skolnick et al (59), T1 lesions were confined to the nasal cavity, T2 and T3 lesions demonstrated progressive involvement of the paranasal sinuses, and T4 lesions extended into the orbit or intracranial cavity.

In each of these systems described for use with IP, a fourstage approach was proposed, with disease isolated to the nose staged as T1, independent of the extent of the disease within the nose. In each system, the involvement of structures outside of the nose and sinuses such as the brain or orbit was staged as T4. Intermediate lesions were staged as either T2 or T3, with authors differing on the assignment of the levels based on the varying involvement of the sinuses.

In all the staging systems, the minimal extent of disease is graded as a lesser stage than diffuse tumor involvement. Furthermore, disease that fills the sinuses diffusely is graded as more advanced.

Krouse established a staging system which would help to segregate patients into easily definable categories (63). The four categories are based on the extent and location of the disease. The described staging system uses CT imaging of the nose and paranasal sinuses and endoscopic examination to determine a certain stage of disease. T1 lesions were confined to the nasal cavity, without extension into the sinuses. The tumor can be localized to one wall or region of the nasal cavity without extension into the sinuses or extranasal compartments. Stages T1 to T3 do not involve concurrent malignancy. Stage T4 involves all tumors with any extranasal/extrasinus extension to adjacent structures but also all tumors associated with malignancy.

Apart from the staging system published by Krouse which is based on extent, location, and malignant association, Han et al used a four-tier categorization (64). This categorization is primarily based on the location and extension of IP and is further focused in planning surgical approaches. Group I involves IP which can be endoscopically removed. Group II requires an adjunctive transantral approach due to exposure to the lateral and anterior maxillary sinus. IPs in group III involve the frontal sinus and additional non-endoscopic procedures are necessary to remove the tumor. In group IV, a craniotomy, orbitotomy, or other open approaches may be required if the extranasal component of the tumor cannot be adequately visualized and resected endoscopically.

Krouse developed a staging system based on the extent of tumor involvement depending on endocsopic, CT and MRI examinations. Han et al set up a categorization which is focused on planning surgical and endoscopic procedures. 


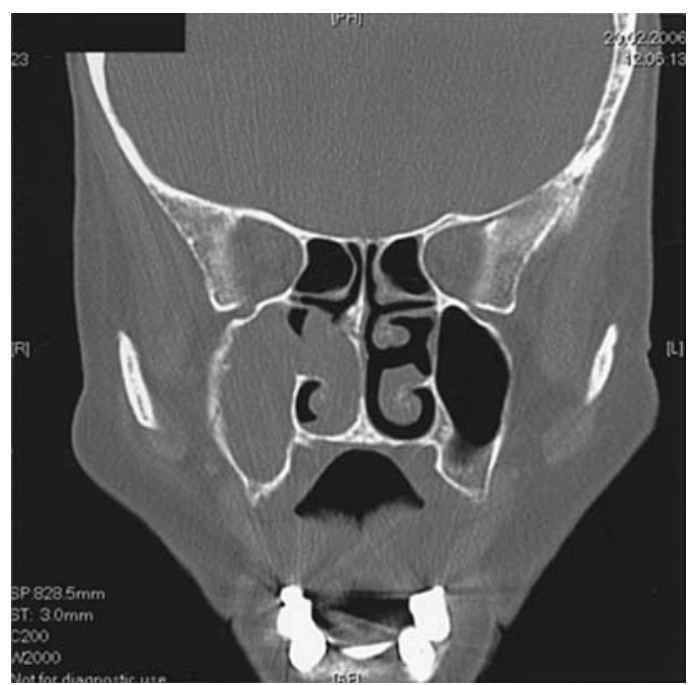

Figure 2. CT scan of an inverted papilloma showing polypoid masses in the left maxillary sinus and adjacent paranasal sinuses as the frequent sites of origin.

Kamel et al recently introduced a new classification system of IP based on the origin of the lesion (65). In type I the tumor originates from the nasal septum or the lateral nasal wall whereas in type II it originates from the maxillary sinus. Kamel claims that with recent advances in functional endoscopic sinus surgery (FESS) IP could be traced to its origin. Furthermore, type I tumors result in nasal obstruction and early diagnosis while the lesion is still small, whereas type II lesions result in late diagnosis with broader extension (66). In terms of recurrence, Kamel et al agree with other authors that recurrence always occurs at the original site due to the incomplete removal of the primary lesion $(6,7,67,68)$. Kamel et al recommend this new classification system to all cases of IP regardless of the extent of the tumor. The authors admit that a long experience in FESS is necessary and that in some cases staging is only finalized during surgery.

There is still no widely accepted classification or staging system for IP. Thus, few reports describe recurrence rates as a function of tumor stage. A relationship between advanced stage and a higher incidence of recurrence has not yet been established $(34,64,69)$.

\section{Macroscopic aspects}

The workup consists of a thorough history and physical exam. The associated symptoms are classically those of unilateral nasal obstruction of varied duration. The patient may have a history of facial pain, rhinorrhea, sinusitis or epistaxis. These are all quite rare. On review, the lateral wall of the nasal fossa and the maxillary sinus are the most frequent sites of origin for IP, whereas its exclusive localization to the frontal $(26,70)$ or sphenoid sinus $(71,72)$ is exceedingly rare and, when involved, is usually caused by an invasion from adjacent sites. Likewise, the intracranial invasion of IP is a rare event, which has been mostly noted at the level of the cribriform plate or the ethmoid roof area in recurrent lesions (73). Intraorbital extension can be observed in lesions with extensive ethmoid involvement. However, the tumor

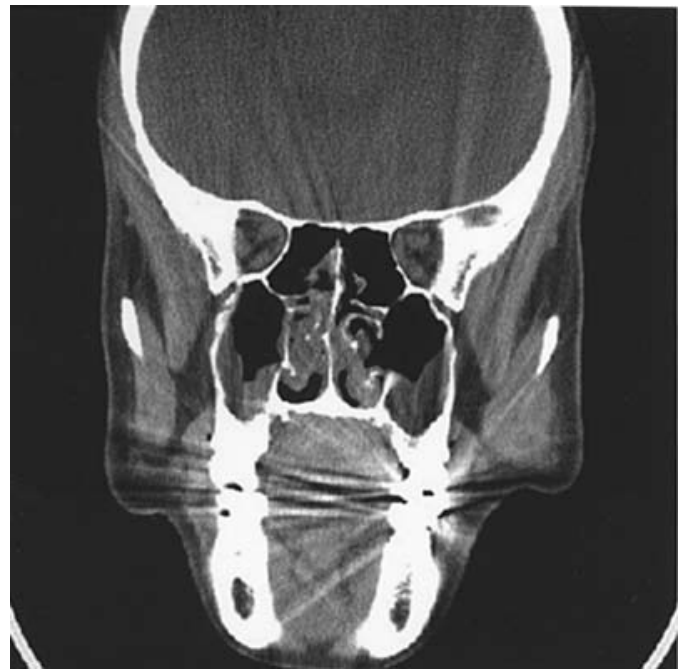

Figure 3. Inverted papilloma arising from the right inferior turbinate of the lateral nasal wall.

usually displaces the orbital content laterally without transgressing the periorbit $(74,75)$.

The presentation of IP is generally unilateral, but the bilateral involvement of the sinonasal tract has been reported in a percentage of patients ranging from <1\% (76) to $9 \%$ (35).

The endoscopic appearance of IP, commonly showing one or more polypoid masses with multiple digitations and a papillary surface located laterally to the middle turbinate, is suggestive of the diagnosis. A CT scan is essential in the evaluation of the tumor (Fig. 2). This allows for the proper surgical approach and extent to be performed. Nevertheless, bony walls; less commonly, sclerotic bony changes can be seen (77-79). However, the CT findings commonly observed in IP are highly aspecific. According to Ojiri et al (80) this limitation can be overcome by using MRI, which, apart from differentiating neoplastic tissue from inflammatory changes, identifies a convoluted cerebriform pattern suggestive of IP on $\mathrm{T} 2$ or enhanced T1-wieghted sequences in about $80 \%$ of cases. If there is any possibility of skull base involvement or vascular tumor by imaging, further radiological studies should be performed such as an MRI or an angiogram. However, an MRI is unable to differentiate the foci of a concomitant malignant neoplasm from IP.

A biopsy of the specimen is necessary to obtain a definitive diagnosis. All nasal biopsies must be taken seriously because of the chance of severe epistaxis or biopsy of the brain. The biopsy should be performed in a controlled setting such as the operating room.

\section{Surgical advances}

There is no doubt that surgery is the treatment of choice, combined with radiotherapy in cases of associated malignancy (35). However, the best surgical approach and the extent of resection are somewhat controversial, as is discussed in the literature (34). Since most sinonasal papillomas arise from the lateral nasal wall, procedures involving a medial maxil- 
lectomy have resulted in the best outcome (35) (Fig. 3). This procedure can be performed with similar results by an endonasal approach or the previously preferred lateral rhinotomy, as shown in various studies $(6,23,28,35,66,67)$. Many authors believe that complete resection is essential for the adequate management and long-term control of IP. Since most sinonasal papillomas arise from the lateral nasal wall, procedures involving a medial maxillectomy have resulted in the best outcome (35). Therefore, for decades, the traditional approach to this lesion has been a lateral rhinotomy or sublabial degloving approach and a medial maxillectomy (81).

Medial maxillectomy through lateral rhinotomy incision is the gold standard for the removal of IP. It has the advantages of the excellent exposure of the lateral nasal wall and paranasal sinuses. The success is related to the en bloc resection of the lateral nasal wall, ethmoid labyrinth, and medial portion of the maxilla which are the sites of formation and extension of this tumor (82). This is a procedure that allows the visualization of the tumor margins while still allowing the preservation of the orbital rim, the eye and its attachments, the lacrimal apparatus, the nasal pyramid, and the palate. Medial maxillectomy allows the en bloc removal of the ethmoidal labyrinth and the medial aspect of the maxilla from the cribriform plate superiorly, to the floor of the nose inferiorly, and from the anterior extent of the ethmoidal cells back to the area of the optic nerve. The lamina papyracea is included in the tissue block. This technique can be expanded to involve the removal of the cribriform plate when combined with an intracranial approach. A lateral rhinotomy incision is made, beginning in the medial aspect of the eyebrow, angling around to midway up the lateral wall of the nose and into the alar groove. A notch can be made in the medial canthal area to prevent webbing. The exposure should be adequate without cutting the lip which also leaves a better final cosmesis. A subperiosteal dissection is performed exposing the anterior wall of the maxillary sinus. The infraorbital nerve is identified and protected. The medial wall of the orbit is dissected exposing the anterior and posterior ethmoid artery which will be the most superior aspect of the dissection. The lacrimal sac is dissected out of its sulcus and is divided at its most distal aspect. An antrostomy in to the maxillary sinus is performed and then the remainder of the maxillary sinus is removed taking care to preserve the infraorbital nerve.

A lateral osteotomy of the nasal bone is performed to give better visual exposure. The first major cut is along the floor of the sinus. This cut is made in the inferior meatus from the anterior tip of the inferior turbinate to the most posterior aspect. The second bone cut entails the most medial part of the orbital rim which is drilled down into the floor of the orbit using a cutting bur. This aspect can be omitted but it can give better visual access with minimal structural defect. The third cut is made along the anterior aspect of the maxillary sinus involving the lacrimal fossa, anterior to the middle turbinate and into the ethmoid cells. The anterior bony rim forming the piriform aperture and the nasal rim are left intact. If the lacrimal duct is left in place the cut is made posterior to it. It is usually cut and marked for stenting later on in the surgery. The fourth cut involves retracting the orbital contents to expose the frontoethmoidal suture line and the anterior ethmoid artery. A small osteotome is used to perforate the ethmoidal cells and the nasal cavity is entered inferior to the suture line, beginning anteriorly in the lacrimal fossa and extending posteriorly.

The suture line and ethmoidal arteries establish the position of the cribriform plate. If a more posterior dissection is required the anterior ethmoid artery can be ligated. The fifth cut involves freeing the posterior and lateral aspect of the lamina papyracea. The cut is extended along the posterior part of the lamina with a curved mayo scissors and goes along the inferior part of the orbit along the rim medial to the infraorbital nerve. This will then join with the drilled incision through the rim. The remaining bony attachment of the lateral nasal wall is that portion of the palatine bone that is anterior to the pterygoid process of the sphenoid bone. This attachment extends from the nasal floor up to the superior turbinate. The en bloc specimen is gently rocked bimanually to reveal the remaining attachments. This is performed using a right-angled scissors starting interiorly through the nose placing the lateral blade into the maxillary sinus while the medial blade lies in the inferior meatus. The curved scissors will make a cut anterior to the pterygoid plate which is the posterior aspect of the inferior and middle turbinates. The superior aspect of the incision is technically impossible to perform using the scissors.

In the era when transnasal resection without endoscopic or microscopic assistance was the most commonly used technique, the percentage of 'recurrences' ranged from $40 \%$ (83) to $78 \%(84)$. We concur with many authors $(7,85)$ that most of these 'recurrences' are basically 'residual' lesions as the exposure offered by the transnasal approach did not guarantee the adequate radicality of the resection. However, although the frequency of recurrence has been lowered, reportedly from $0 \%$ (35) to $29 \%$ (86), this technique has been associated with complications such as epiphora, chronic dacryocystitis, transient diplopia, and eustachian tube dysfunction. An average hospitalization time of 7 days and possible esthetic sequelae related to the facial scar were also present $(1,86)$. With the intent to avoid the latter concern, other techniques not requiring facial incisions such as RougeDenker's surgery (87), septal translocation (88), and midfacial degloving (89) have been used.

The local aggressiveness of IP has caused a change in management from the lateral rhinotomy to other external approaches. Midfacial degloving is an excellent alternative open procedure. It also provides excellent bilateral exposure and does not require an external scar. The midfacial degloving procedure utilizes a combination of four facial incisions with or without osteotomies of the nasal bone and frontal process of the maxilla. In this technique four incisions are made: i) A bilateral sublabial incision, ii) a complete transfixion between the columella and septum, iii) bilateral piriform aperture incisions extending to the vestibule, and iv) either an intercartlaginous or marginal incision (used with an external rhinoplasty). It is best to overlap at right angles at the corners to prevent rounding and web formation. The lower framework of the nose is released similar to the septorhinoplasty approach. The periosteum of the maxilla is elevated preserving the infraorbital nerves. The skin, and lower $1 / 3$ of the nose can be elevated to the glabella and orbit. This is secured and retracted with a penrose through the nostrils. The internal 
maxillary artery may be encountered and ligated as it enters the pterygopalatine fossa. Sachs et al, in their series of 46 patients, offered the midfacial degloving approach, as it improves the visibility of the total surgical field (89). Results of these techniques have been more acceptable with recurrence rates in the 3 to $13 \%$ range (89). Similarly, Price et al described the use of the midfacial degloving approach, although a follow-up of the patients was not given (90). As an alternative to lateral rhinotomy Esteban et al recommended midfacial degloving as the procedure of choice for block medial maxillectomy and ethmoidectomy (91), compared to Lawson et al who reported that lateral rhinotomy and medial maxillectomy were the gold standard for the majority of cases (6). A study by Yoskovitch et al stated that more aggressive surgery is associated with a more definitive treatment and significantly less recurrence, as recurrence was seen in $45 \%$ of patients treated conservatively by local excision compared with no recurrence in patients treated aggressively with lateral rhinotomy or medial maxillectomy (92).

The disadvantages to this approach are: i) The potential for nasal vestibular stenosis, and ii) difficulty with superior ethmoid exposure in large tumors. Vestibular stenosis can be avoided with a proper incision design. Other complications include oroantral fistula, epistaxis, and nasal crusting which are present with the medial maxillectomy as well as midfacial degloving. The advantage is avoidance of a facial scar and allowing bilateral exposure.

The precise identification of lesions by modern imaging techniques such as CT and MRI and the excision of intranasal lesions with endoscopes has led to the application of these procedures in the treatment of IP. The first report on the endoscopic treatment of IP dates back to 1981, when Stammberger documented 15 patients treated by a purely endsoscopic approach (93). Buchwald et al reported that the endoscopic surgery of IP can be supplemented by a midfacial degloving procedure or a lateral rhinotomy if the tumor cannot be visualized sufficiently by an endoscopy (8). Waitz and Wigand reported on a series of 35 patients with a mean follow-up of 46 months (67). They reported a 19\% recurrence rate with the extranasal approach and $17 \%$ with the endoscopic approach. This study could be biased however, due to the selection of very small lesions with no evidence of malignancy. These lesions were less aggressive with a lower overall final malignancy rate of $4 \%$ compared to averages of about $11 \%$ in other literature. Lawson et al compared various aspects of IP management, such as treatment concepts and surgical approaches in 160 patients with an average followup period of 5.2 years (33). Endoscopic removal can be performed on selected lesions with a recurrence rate of $12 \%$ compared to that of more aggressive techniques of $18 \%$ (33). Pasquini et al compared traditional and endoscopic methods in 89 cases with a follow-up period of 96 months and 54 months, respectively (94). They reported a lower recurrence rate of $3 \%$ and a mean hospital stay of 1 day with endoscopic approaches compared to a $24 \%$ recurrence rate and 5 days hospitalization with traditional methods. Pasquini et al concluded that Krouse stages T1, T2, and select T3 cases are all suitable for endoscopic resection. Tomenzoli et al reported the accurate removal of IP by different endoscopic strategies in 47 patients during an 8-year period. No recurrences were observed after a mean follow-up of 55 months (69). Kaza and Casiano reported 51 cases of IP being removed endoscopically over a 10-year period (95). They reported a recurrence rate of $14 \%$ with a mean follow-up of 30 months. The authors recommend that the extent of resection should be based on the intraoperative endoscopic findings, and not necessarily on the preoperative CT or MRI findings. Kraft et al reported 17 cases of IP with a recurence rate of $11.7 \%$ and a mean follow-up of 62 months (96). Busquets and Hwang treated 28 patients endoscopically with a recurrence rate of $10 \%$ with a mean follow-up of 22 months (97). Complications of FESS for IP have been reported between 0 and $19.6 \%$ $(33,69,94,96)$. Most were minor complications, such as epistaxis, epiphora, temporary infraorbital hypesthesia, minimal orbital fat exposure and so on. Cerebral spinal fluid leakage was reported in $<6 \%$ of cases (95).

It is extremely difficult to compare the results obtained with external vs endoscopic or micro-endoscopic procedures due to the extreme variability in preoperative assessment, the extent of disease, and the follow-up length in the different studies reported. A follow-up period of at least two years should be mandatory in order to compare the approaches as most recurrent lesions are diagnosed 24 months after surgery $(64,82,86)$. Another important fact is the lack of an officially recognized staging or classification system although several systems have been reported by Krouse (63), Han (64) and Kamel (65).

Radiation is not usually recommended instead of surgery. It has been suggested that it could be effective in the treatment of advanced aggressive tumors or in patients who are poor surgical candidates. There has been some concern with the use of radiation therapy for the treatment of IP without an associated malignancy. A study by Gomez et al reported 4 out of 14 patients treated for benign disease with radiation therapy (98). Two other studies by Weissler et al and Mendenhall et al have shown no conversion to malignancy and excellent control rates $(35,99)$. Combined surgical and radiation therapy is effective in patients found to have SCCA associated with their IP.

To our knowledge, no investigation so far has emphasized the importance of a preoperative medical protocol. Systemic antibiotics and corticosteroids can reduce concomitant inflammatory polyps, affording a clearer picture of the extent of disease and eventually the site of origin. In addition, they significantly diminish bleeding during the surgical procedure (100). Hence, radiological investigations without the abovementioned protocol could result in an overestimation of the tumor extent. This can lead the surgeon to a more aggressive surgery than necessary (82).

Recently, however, new concepts in the pathogenesis of IP have been raised by Roh et al (101). They suggest that IP could be the end-stage of a chronic inflammatory condition, and not a neoplasm. Therefore, conservative surgical treatment with a close follow-up and careful review of the final pathological specimen would be more appropiate.

With some of the new data suggesting that HPV could be a potential aetiologic factor in the development of IP as described in 'Cytogenetics and molecular genetics', the use of antiviral agents could be beneficial. Interferon has been suggested for the use of a patient with multiple recurrences, 
advanced disease, or spread to the orbit and skull base. This is still under investigation.

\section{Conclusion}

Unilateral nasal obstruction or unilateral nasal polyps should alert the otolaryngologist to the possibility of IP. IP represents a benign neoplastic proliferation with a high recurrence rate. It has the propensity for invasion into adjacent structures, such as the orbit and CNS even in the absence of malignancy. The tumor is classified as an 'intermediate' tumor and should be handled by an en bloc surgical approach. Its presumed neoplastic nature and putative role as a precursor to squamous cell carcinoma have not been comfirmed at the molecular genetic level. IPs are monoclonal proliferations and yet they do not fit the profile of a prototypic precursor lesion. There is still evidence that HPV infection can be an early event in the multistep process in the malignant transformation of IP. An increased EGFR and TGF- $\alpha$ expression is associated with early events in IP carcinogenesis. The recurrence rate is related to the adequacy of the surgical excision. A simple excision with a polypectomy or the Caldwell Luc approach resulted in 67 to $78 \%$ recurrence, whereas when lateral rhinotomy and medial maxillectomy were performed, recurrence was 0 to $14 \%$. Even this is quite high for a benign disease. Therefore, it could be related to a multicentric disease process. Aggressive first-time surgical management aiming at the removal of the lesion together with a margin of normal mucosa should be the aim of the therapy. The lateral rhinotomy approach with a medial maxillectomy and wide excision of the lesion is satisfactory in managing larger lesions. The advances in endonasal micro-endoscopic surgery allow both the safe and effective removal of IP with low morbidity, and therefore it should be the first choice approach. The propensity for delayed recurrences and the up to $13 \%$ incidence of malignant transformation mandates careful, long-term followup.

\section{References}

1. Bielamowicz S, Calcaterra TC and Watson D: Inverting papilloma of the head and the neck: UCLA update. Otolaryngol Head Neck Surg 109: 71-76, 1993.

2. Ward N: A mirror of the practice of medicine and surgery in the hospitals of London: London Hospital. Lancet 2: 480-482, 1854.

3. Billroth T: Ueber dem Bau der Schleimpolyp. G. Reimer, Berlin, p11, 1855.

4. Kramer R and Som ML: True papilloma of the nasal cavity. Arch Otolaryngol Head Neck Surg 22: 22-25, 1935.

5. Ringertz N: Pathology of malignant tumours arising in the nasal and paranasal cavities and maxilla. Acta Otolaryngol Suppl 27: 31-42, 1938.

6. Lawson W, Ho BT, Shaari CM and Biller HF: Inverted papilloma: a report of 112 cases. Laryngoscope 105: 228-288, 1995.

7. Hyams VJ: Papillomas of the nasal cavity and paranasal sinuses: a clinicopathological study of 315 cases. Ann Otol Rhinol Laryngol 80: 192-206, 1971

8. Buchwald C, Franzmann M-B and Tos M: Sinonasal papillomas: A report of 82 cases in Copenhagen County, including a longitudinal epidemiological and clinical study. Laryngoscope 105: 72-79, 1995.

9. Seshul MJ, Eby TL, Crowe DR and Peters GE: Nasal inverted papilloma with involvement of middle ear and mastoid. Arch Otolaryngol Head Neck Surg 121: 1045-1048, 1995.

10. Cooter MS, Charlton SA, Lafreniere D and Spiro J: Endoscopic management of an inverted nasal papilloma in a child. Otolaryngol Head Neck Surg 118: 876-879, 1998.
11. Minovi A, Kollert M, Draf W and Bockmühl U: Endonasal micro-endoscopic resection of sinonasal inverted papilloma. Laryngorhinootologie 85: 421-425, 2006.

12. Bura M, Vladika I, Aralica G, Bumber Z, Krajina Z, Bijelic L, Poje G, et al: Inverted sinonasal papilloma - a report of 31 cases and review of the literature. Coll Antropol 27: 271-278, 2003.

13. Thorp MA, Oyarzabal-Amigo MF, du Plessis JH and Sellars SL: Inverted papilloma. Laryngoscope 111: 1401-1405, 2001.

14. Klimek T, Atai E, Schubert M and Glanz H: Inverted papilloma of the nasal cavity and paranasal sinuses: clinical data, surgical strategy and recurrence rates. Acta Otolaryngol 120: 267-272, 2000.

15. Mansell NJ and Bates GJ: The inverted schneiderian papilloma: a review and literature report of 43 new cases. Rhinology 38: 97-101, 2000.

16. Tang AC, Grignon DJ and MacRae DL: The association of human papillomavirus with Schneiderian papillomas: a DNA in situ hybridization study. J Otolaryngol 23: 292-297, 1994.

17. Wong CJW, Gallagher GJ, Gertz R, Chang ALC and Shklar G: TGF-alpha in chemically transformed hamster oral keratinocytes. Cancer Res 48: 3130-3134, 1988.

18. Grandes JR and Tweardy DJ: Elevated levels of transforming growth factor receptor messenger RNA are early makers of carcinogenesis in head and neck cancer. Cancer Res 53: 3579-3584, 1993.

19. Brandwein M, Steinberg B, Thung S, Biller H, Dilorenzo T and Galli R: Human papillomavirus 6/11 and 16/18 in Schneiderian inverted papillomas. In situ hybridization with human papillomavirus RNA probes. Cancer 63: 1708-1713, 1989.

20. Furuta Y, Shinohara T, Sano K, Nagashima K, Inoue K, Tanaka K and Inuyama Y: Molecular pathologic study of human papillomavirus infection in inverted papilloma and squamous cell carcinoma of nasal cavities and paranasal sinuses. Laryngoscope 101: 79-85, 1991.

21. Gaffey MJ, Frierson HF, Weiss LM, Barber CM, Baber GB and Stoler MH: Human papillomavirus and Epstein-Barr virus in sinonasal Schneiderian papillomas. An in situ hybridization and polymerase chain reaction study. Am J Clin Pathol 106: 475-482, 1996.

22. Beck JC, McClatchey KD, Lesperance MM, Esclamado RM, Carey TE and Bradford CR: Human papillomavirus types important in progression of inverted papilloma. Otolaryngol Head Neck Surg 113: 558-563, 1995.

23. Vrabec DP: The inverted schneiderian papilloma: A clinical and pathological study. Laryngoscope 85: 186-220, 1975.

24. Weber RS, Shillitoe EJ, Robbins TK, Luna MA, Batsakis JG, Donovan DT and Adler-Storthz K: Prevalence of human papillomavirus in inverted nasal papillomas. Arch Otolaryngol Head and Neck Surg 114: 23-26, 1988.

25. Buchwald C, Franzmann MB, Jacobsen GK, Juhl BR and Lindeberg H: Carcinomas occuring in papillomas of the nasal septum associated with human papillomavirus (HPV). Rhinology 35: 74-78, 1997.

26. Chee LW and Sethi DS: The endoscopic management od sinonasal inverted papillomas. Clin Otolaryngol 24: 61-66, 1999.

27. McCary WS, Gross CW, Reibel JF and Cantrell RW: Preliminary report: endoscopic versus external surgery in the management of the inverting papilloma. Laryngoscope 104: 415-419, 1994.

28. Stankiewicz JA and Girgis SJ: Endoscopic surgical treatment of nasal and paranasal sinus inverted papilloma. Otolaryngol Head Neck Surg 109: 988-995, 1993

29. Tufano RP, Thaler ER, Lanza DC, Goldberg AN and Kennedy DW: Endoscopic management of sinonasal inverted papilloma. Am J Rhinol 13: 423-426, 1999.

30. Yamaguchi KT, Shapsay SM and Incze JS: Inverted papilloma and squamous cell carcinoma. J Otolaryngol 8: 171-178, 1979.

31. Kapadia SB, Barnes L, Pelzman K, Mirani N, Heffner DK and Bedetti C: Carcinoma ex oncocytic Schneiderian (cylindrical cell) papilloma. Am J Otolaryngol 14: 332-338, 1993.

32. Ridolfi RL, Lieberman PH, Erandson RA and Moore OS: Schneiderian papillomas: a clinicopathological study of 30 cases. Am J Surg Pathol 1: 43-53, 1977.

33. Lawson W, Kaufman MR and Biller HF: Treatment outcomes in the management of inverted papilloma: an analysis of 160 cases. Laryngoscope 113: 1548-1556, 2003.

34. Krouse JH: Endoscopic treatment of inverted papilloma: safety and efficacy. Am J Otolaryngol 22: 87-99, 2001.

35. Weissler MC, Montgomery WW, Turner PA, Montgomery SK and Joseph MP: Inverted papilloma. Ann Otol Rhinol Laryngol 95: 215-221, 1986. 
36. Fechner RE and Alford DO: Inverted papilloma and squamous carcinoma. An unusual case. Arch Otolaryngol 88: 507-512, 1968.

37. Gould VE, Manosca F, Reddy VB and Gattuso P: Cytologichistologic correlations in the diagnosis of inverted sinonasal papilloma. Diagn Cytopathol 30: 201-207, 2004

38. Gerdes J, Lemke H, Baisch H, Wacker HH, Schwaab U and Stein H: Cell cycle analysis of a cell proliferation associated human nuclear antigen defined by the monoclonal antibody Ki67. J Immunol 133: 1710-1715, 1984.

39. Syrjanen KJ: HPV infections in benign and malignant sinonasal lesions. J Clin Pathol 56: 174-181, 2003.

40. Bergler W and Götte K: Current advances in the basic research and clinical management of juvenile-onset recurrent respiratory papillomatosis. Eur Arch Otolaryngol 257: 263-269, 2000.

41. Hwang CS, Yang HS and Hong MK: Detection of HPV in sinonasal inverted papillomas using polymerase chain reaction (PCR). Am J Rhinol 12: 363-366, 1998.

42. Katori H, Nozawa A and Tsukuda M: Markers of malignant transformation of sinonasal inverted papilloma. Eur J Surg Oncol 31: 905-911, 2005.

43. Kashima HK, Kessis T, Hruban RH, Wu TC, Zinreich SJ and Shah KV: Human papillomavirus in sinonasal papillomas and squamous cell carcinoma. Laryngoscope 102: 973-976, 1992.

44. Nagao S, Yoshinouchi M, Miyagi Y, Hongo A, Kodama J, Itoh S and Kudo T: Rapid and sensitive detection of physical status of human papillomavirus type 16 by quantitative real-time PCR. J Clin Microbiol 40: 863-867, 2002.

45. McKay SP, Gregoire L, Lonardo F, Reidy P, Mathog RH and Lancaster WD: Human papillomavirus (HPV) transcripts in malignant inverted papilloma are from integrated HPV DNA. Laryngoscope 115: 1428-1431, 2005.

46. Scheffner M, Romanczuk H, Munger K, et al: Functions of human papillomavirus proteins. Curr Top Microbiol Immunol 186: 83-99, 1994

47. Lukas J, Muller H, Bartkova J, et al: DNA tumor virus oncoproteins and retinoblastoma gene mutations share the ability to relieve the cell's requirement for cyclin D1 function in G1. J Cell Biol 125: 625-638, 1994.

48. Malanchi I, Caldeira S, Krutzfeldt M, Giarre M, AlunniFabbroni $\mathrm{M}$ and Tommasino M: Identification of a novel activity of human papillomavirus type E16 protein in deregulating the G1/s transition. Oncogene 21: 5665-5672, 2002.

49. Zerfass-Thome K, Zwerschke W, Mannhardt B, Tindle R, Botz JW and Jansen-Duerr P: Inactivation of the cdk inhibitor $\mathrm{p} 27^{\mathrm{KIP} 1}$ by the human papillomavirus type E7 oncoprotein. Oncogene 13: 2323-2330, 2002

50. Affolter A, Helmbrecht S, Finger S, Hörmann K and Götte K: Altered expression of cell cycle regulators p21, p27 and p53 in tumors of salivary glands and paranasal sinuses. Oncol Rep 13: 1089-1094, 2005

51. Saegusa M, Nitta H, Hashimura M and Okayasu I: Downregulation of $\mathrm{p} 27^{\mathrm{KIP} 1}$ expression is correlated with increased cell proliferation but not expression of $\mathrm{p} 21^{\mathrm{WAF} 1}$ and $\mathrm{p} 53$, and human papillomavirus infection in benign and malignant tumours of sinonasal regions. Histopathology 35: 55-64, 1999.

52. Gusterson BA, Anbazhagan R, Warren W, et al: Expression of p53 in premalignant and malignant squamous epithelium. Oncogene 6: 1785-1789, 1991.

53. Union Internationale contre le Cancer: TNM Classification of Malignant Tumours. 6th edition. Wiley, New York, 2002

54. Cantu G, Solero CL, Mariani L, Mattavelli F, Pizzi N and Licitra L: A new classification for malignant tumors involving the anterior skull base. Arch Otolaryngol Head Neck Surg 125: 1252-1257, 1999

55. American Joint Committee on Cancer: Manual for Staging of Cancer. Lippincott-Raven, Philadelphia, 1997.

56. Carinci F, Curioni C, Padula E and Calearo C: Cancer of the nasal cavity and paranasal sinuses: a new staging system. Int $\mathrm{J}$ Oral Maxillofac Surg 25: 34-39, 1966.

57. Friedman WH, Katsantonis GP, Sivore M and Kay S: Computed tomography staging of the paranasal sinuses in chronic hyperplastic rhinosinusitis. Laryngoscope 100: 1161-1165, 1990.

58. Lund VJ and MacKay IS: Staging in rhinosinusitis. Rhinology 31: 183-184, 1993.

59. Skolnick EM, Loewy A and Friedman JE: Inverted papilloma of the nasal cavity. Arch Otolaryngol 64: 83-89, 1966.

60. Norris HJ: Papillary lesions of the nasal cavity and paranasal sinuses, II: inverting papilloma - a study of 29 cases. Laryngoscope 73: 1-17, 1963.
61. Schneider D: A new staging system for the inverted papilloma of the paranasal sinuses. Laryngorhinootologie 55: 894-896, 1976

62. Schwab W, Schmeisser KJ, Steinhoff HJ and Mang WL: Instructions for the documentation of neoplasms in the area of the head and neck following the TNM system. HNO 33: 337-348, 1985.

63. Krouse JH: Development of a staging system for inverted papilloma. Laryngoscope 110: 965-968, 2000.

64. Han JK, Smith TL, Loehrl T, Toohill RJ and Smith MM: An evolution in the management of sinonasal inverting papilloma. Laryngoscope 111: 1395-1400, 2001.

65. Kamel RH, Khaled A and Kandil T: Inverted papilloma: New classification and guidelines for endoscopic surgery. Am J Rhinol 19: 358-364, 2005.

66. Kamel RH: Transnasal endoscopic medial maxillectomy in inverted papilloma. Laryngoscope 105: 847-853, 1995.

67. Waitz G and Wigand ME: Results of endoscopic sinus surgery for the treatment of inverted papillomas. Laryngoscope 102: 917-922, 1992.

68. Sham CL, Woo JK and van Hasselt CA: Endoscopic resection of inverted papilloma of the nose and paranasal sinuses. J Laryngol Otol 112: 758-764, 1998.

69. Tomenzoli D, Castelnuovo P, Pagella F, et al: Different endoscopic surgical strategies in the management of inverted papilloma of the sinonasal tract: experence with 47 patients. Laryngoscope 114: 193-200, 2004.

70. Shohet JA and Duncavage JA: Management of the frontal sinus with inverted papilloma. Otolaryngol Head Neck Surg 114: 649-652, 1996.

71. Yiotakis I, Psarommatis I, Manolopoulos L, Ferekidis E and Adamopoulos G: Isolated inverted papilloma of the sphenoid sinus. J Laryngol Otol 115: 227-230, 2001.

72. Lee JT, Bhuta S, Lufkin R and Castro DJ: Isolated inverting papilloma of the sphenoid sinus. Laryngoscope 113: 41-44, 2003.

73. Vural E, Suen JY and Hanna E: Intracranial extension of inverted papilloma: an unusual and potentially fatal complication. Head Neck 21: 703-706, 1999.

74. Elner VM, Burnstine MA, Goodman ML and Dortzbach RK: Inverted papillomas that invade the orbit. Arch Ophthalmol 113: 1178-1183, 1995.

75. Bajaj MS and Pushker N: Inverted papilloma invading the orbit. Orbit 21: 155-159, 2002.

76. Phillips PP, Gustafson RO and Facer GW: The clinical behaviour of inverting papilloma of the nose and paranasal sinuses: report of 112 cases and review of the literature. Laryngoscope 100: 463-469, 1990.

77. Lund VJ and Lloyd GA: Radiological changes associated with inverted papilloma of the nose and paranasal sinuses. Br J Radiol 57: 455-461, 1984.

78. Woodruff WW and Vrabec DP: Inverted papilloma of the nasal vault and paranasal sinuses: spectrum of CT findings. Am J Roentgenol 162: 419-423, 1994.

79. Dammann F, Pereira P, Laniado M, Plinkert P, Lowenheim H and Claussen CD: Inverted papilloma of the nasal cavity and the paranasal sinuses: using CT for primary diagnosis and followup. Am J Roentgenol 172: 543-548, 1999.

80. Ojiri H, Ujita M, Tada S and Fukuda K: Potentially distinctive features of sinonasal inverted papilloma on MR imaging. Am J Roentgenol 175: 465-468, 2000

81. Cody DT II: Neoplasm of the nasal cavity. In: Otolaryngology Head \& Neck Surgery. Cumming CWFJ, Harker LA and Krause CJ (eds). Mosby-Year Book Inc., pp883-901, 1998.

82. Sukenik MA and Casiano R: Endoscopic medial maxillectomy for inverted papillomas of the paranasal sinuses: value of the intraoperative endoscopic examination. Laryngoscope 110: 39-42, 2000.

83. Oberman HA: Papillomas of the nose and paranasal sinuses: Am J Clin Pathol 42: 254-258, 1964.

84. Calcaterra TC, Thompson JW and Paglia DE: Inverting papillomas of the nose and paranasal sinuses. Laryngoscope 90: 53-60, 1980.

85. Lund VJ: Optimum management of inverted papilloma. J Laryngol Otol 114: 194-197, 2000.

86. Myers EN, Schramm VL and Burns EL: Management of inverted papilloma of the nose and paranasal sinuses. Laryngoscope 91: 2071-2084, 1981

87. Sanderson RJ and Knegt P: Management of inverted papilloma via Denker's approach. Clin Otolaryngol 24: 69-71, 1999.

88. Sofferman RA: The septal translocation procedure: an alternative to lateral rhinotomy. Otolaryngol Head Neck Surg 167: 803-808, 1988 . 
89. Sachs ME, Conley J, Rabuzzi DD, Blaugrund S and Price J: Degloving approach for the total excision of inverted papillomas. Laryngoscope 94: 1595-1598, 1984.

90. Price JC, Holliday MJ and Johns ME: The versatile midface degloving approach. Laryngoscope 98: 291-295, 1988.

91. Esteban F, Jurado A, Cantillo E, Mochon A and Casado JM: Facial degloving as a versatile approach to nasal sinus tumors. Acta Otorhinolaryngol Esp 48: 457-460, 1997.

92. Yoskovitch A, Braverman I, Nachtigal D, Frenkiel S, Rochon L and Black MJ: Sinonasal schneiderian papilloma. J Otolaryngol 27: 122-126, 1998.

93. Stammberger H: Zum invertierten Papillom der Nasenschleimhaut. HNO 29: 128-133, 1981.

94. Pasquini E, Sciarretta V, Farneti G, Modugno GC and Ceroni AR: Inverted papilloma: report of 89 cases. Am J Otolaryngol 25: 178-185, 2004.

95. Kaza S and Casiano RR: Endoscopic resection of inverted papilloma: University of Miami experience. Am J Rhinol 17: 185-190, 2003.
96. Kraft M, Kaufmann T and Holzmann D: Long-term results of endonasal sinus surgery in sinonasal papillomas. Laryngoscope 113: 1541-1547, 2003.

97. Busquets JM and Hwang PH: Endoscopic resection of sinonasal inverted papilloma: a meta-analysis. Otolaryngol Head Neck Surg 134: 476-482, 2006.

98. Gomez JA, Mendenhall WM, Tannehill SP, Stringer SP and Cassisi NJ: Radiation therapy in inverted papilloma in the nasal cavity and paranasal sinuses. Am J Otolaryngol 21: 174-178, 2000.

99. Mendenhall WM, Million RR, Cassisi NJ and Pierson KK: Biologically aggressive papillomas of the nasal cavity: the role of radiation therapy. Laryngoscope 95: 344-347, 1985.

100. Van Camp C and Clement PA: Results of oral steroid treatment in nasal polyposis. Rhinology 32: 5-9, 1994.

101. Roh HJ, Procop GW, Batra PS, Citardi MJ and Lanza DC: Inflammation and the pathogenesis of inverted papilloma. Am J Rhinol 18: 65-74, 2004. 